\title{
水环境中抗生素耐药性的科学研究前沿、 环境健康风险评估和控制阻断策略
}

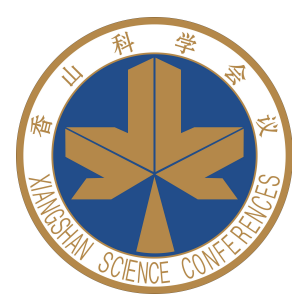

张制 $^{*}$, 李炳 ${ }^{2}$

1. 香港大学土木工程系环境微生物组工程技术实验室, 香港;

2. 清华大学深圳国际研究生院环境工程与管理研究所, 深圳 518055

*联系人, E-mail: zhangt@hku.hk

2020-02-08 收稿, 2020-05-07 修回, 2020-05-08 接受, 2020-05-09 网络版发表

香港研究资助局主题研究计划(T21-604/19-R)、广东省自然科学基金杰出青年项目(2019B151502034)和污染控制与资源化研究国家重点实验 室开放基金(PCRRF17038)资助

摘要 水环境是抗生素耐药性传播的关键节点. 然而, 对水环境中耐药基因的来源、迁移和归趋仍缺乏深入的科学 研究. 对于已具备抗生素耐药基因暴露水平的环境, 如何进行环境健康风险评估仍缺乏基本框架和精准模型. 此外, 控制阻断策略的制订和实施也缺少全局性布局. 本文将聚焦上述三方面, 以水环境为主, 探讨环境抗生素耐药性的 科学研究前沿、环境健康风险评估和控制阻断策略. 科学前沿包括: (1) 耐药基因的主要选择压力——抗生素的标 准检测体系与共享基础数据库的建立; (2) 水环境中抗生素的迁移转化和生物可利用性; (3) 耐药基因在污水处理厂 生态系统中的转移和扩散; (4) 探索耐药基因暴露水平研究的标准化方法: 宏基因组学和高通量qPCR; (5) 耐药基因 水平转移和环境传播的关键可移动遗传元件; (6) 耐药基因的基因组大数据深度挖掘和机器学习. 环境健康风险评 估需区分两种风险，即抗生素治疗失效的风险和耐药基因在环境中传播的风险. 耐药性的防控阻断涉及多学科联 动, 需要临床医学、生态学、农学、药学、环境科学与工程、教育学等多方共同努力。应从“卫生一体化”(One Health)的全局出发, 优先在关键排放源等节点上加大防控投入, 以有效阻断抗生素和耐药基因的环境排放.

关键词 耐药基因, 水环境, 水平转移, 风险评估, 控制阻断

2015年, 世界卫生组织(WHO)在其报告“Global action plan on antimicrobial resistance”中将细菌抗生素耐 药性(抗性)问题列为 21 世纪人类在健康领域面临的最 大挑战之一. 抗生素作为抑菌杀菌的药物, 自 20 世纪 30 年代弗莱明发明青霉素并广泛应用于临床治疗以来, 在人类健康保护、畜牧和农业生产中发挥了无可替代 的作用. 目前使用的抗生素主要包括 $\beta$-内酰胺类、氨 基糖苷类、四环素类、大环内酯类、磺胺类、喹诺酮 类、氯霉素类和糖肽类等. 我国是抗生素生产和使用 大国, 每年的消费总量约为 16.2 万 $\mathrm{t}$, 是美国总用量的近
10倍，英国总用量的 150 倍，其中人用抗生素占到总量 的 $48 \%$, 兽用抗生素为 $52 \%{ }^{[1]}$.

联合国环境规划署发布的“Frontiers 2017, Emerging Issues of Environmental Concern”指出, 抗生素的 滥用已成为严重问题：从2000 2009年，人类抗生素的 消耗量增长了 $36 \%$, 预计到2030年畜牧业的抗生素使用 量将会增长67\%(https://www.unenvironment.org/resources/frontiers-2017-emerging-issues-environmentalconcern). 由于抗生素的过量使用和滥用的加剧, 细菌 耐药性也在不断进化、增强, 甚至出现了能耐受几乎 
所有抗生素的“超级细菌”, 从而使受感染人群面临死 亡风险. 抗生素新药研发乏力的现状与细菌耐药性日 趋严重的“剪刀差”，导致全球范围内已爆发多起因携 带耐药基因的超级细菌感染导致的恶性死亡事件. 由 英国政府委托进行的2014年奥尼尔报告“Review on antimicrobial resistance: Tackling a crisis for the health and wealth of nations”估计: 到2050年, 抗生素耐药性感 染可能成为全球引起死亡的首要原因 ${ }^{[2]}$. 我国的耐药问 题尤为严峻, 据英国抗生素耐药评估委员会预测, 如目 前抗生素滥用的情况得不到改善, 到2050年中国将有 100 万人因耐药菌感染而死亡.

抗生素耐药细菌(ARB, 又称抗性细菌)和抗生素耐 药基因(ARGs, 又称抗性基因)的传播及其对公众健康 的威胁, 近年来已经引起了全世界的广泛关注 ${ }^{[3]}$, 国际 组织和各国政府纷纷制定了针对ARB和ARGs的防控 计划. 2013年6月在伦敦召开的八国集团首脑会议(G8 峰会)明确指出, 抗生素耐药性问题是与全球气候变 暖、水危机同等严峻的全球性危机 ${ }^{[4]}$. 2014年9月美国 政府提出需要从国家战略的高度来应对抗生素抗性 ${ }^{[5]}$. 2015年5月WHO发布全球报告, 呼吁建立全球抗生素抗 药性监测系统(https://www.who.int/antimicrobial-resistance/publications/surveillance-system-manual/en/). 2016 年9 月联合国大会第71次会议上, 联合国各成员国采纳 了抗生素耐药性高级别会议的政治宣言 (https://www. un.org/zh/documents/treaty/files/A-RES-71-3.shtml). 中 国于2016 年8 月由国家卫生和计划生育委员会、国家 发展和改革委员会和环境保护部等14部委联合发布了 《遏制细菌耐药国家行动计划(2016 2020年)》(http:// www.gov.cn/xinwen/2016-08/25/content_5102348.htm), 以期通过多部门协同谋划及联防联控对抗生素耐药菌 和耐药基因的产生与传播实行有效管控.

环境是抗生素耐药性传播的一个关键节点. 土 壤、河流、湖泊、海水中的细菌可因为排放到环境中 的耐药菌、抗生素以及人类使用的其他类化学物质 (如消毒剂、重金属等)而产生耐药性. 而人和牲畜会通 过食物、水和空气接触更多的耐药细菌受到致病菌的 感染，为应对这些感染而使用的抗生素又开始了新一 轮的耐药演化循环. 近年来, 除了对临床抗生素耐药性 的关注之外, 对于进人环境的耐药细菌和耐药基因的 研究也逐渐深人. 目前, 在各种环境中, 包括土壤 ${ }^{[6]}$ 、

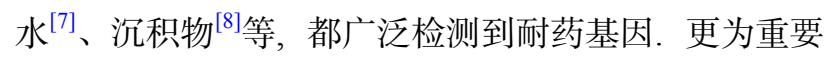
的是这些耐药基因有可能通过水平基因转移(HGT)从
环境宿主细菌转移到病原菌, 或者从病原菌转到环境 中的土著宿主细菌. 联合国环境规划署发布的“Frontiers 2017, emerging issues of environmental concern” 提出, 当前全球六大新兴环境问题中, 抗菌剂耐药性: 环境调查位列第一(https://www.unenvironment.org/resources/frontiers-2017-emerging-issues-environmentalconcern), 希望引起对它们环境赋存状态与传播行为的 更广泛关注，进而评估其环境健康风险并研讨防控阻 断策略.

目前普遍认为耐药基因的环境传播介质主要为水 (污水处理系统和自然水体)、土壤和空气. 水环境因具 有较大的流动性和较高的生物量而受到更为广泛的关 注. 医院废水、居民生活污水经污水收集管网排放至 污水处理厂, 是耐药菌群和耐药基因的重要点源污染 源. 多项研究表明, 污水处理厂中进水、活性污泥及出 水中都含有相当丰度的耐药基因 ${ }^{[9]}$. 随着污水处理系统 的出水排放至自然水体, 如海洋、湖泊和河流, 连同部 分畜禽养殖及农业废水的耐药基因的面源污染汇人， 使自然水体成为了耐药菌群和耐药基因的重要传播载 体. 研究人员分别在河水、底泥, 甚至饮用水中发现了 多样的耐药基因和耐药细菌 ${ }^{[8,10]}$. 这些研究初步探明了 水环境中耐药基因的存在类型、多样性和污染分布状 况，为后续布点采样继而进行耐药基因的环境追踪与 溯源研究提供了有效支撑.

然而，对环境中耐药基因的来源、迁移和归趋仍 缺乏系统和深人的科学研究. 对于已经具备一些基础 数据和抗生素耐药基因暴露水平的环境, 如何进行环 境健康风险评估仍然缺乏基本框架和精准模型. 对控 制阻断策略的制定和实施方案也缺少全局性的思考和 研讨. 本文将针对上述三方面的问题，以水环境为主， 结合其他环境, 探讨环境抗生素耐药性的科学研究前 沿、环境健康风险评估和控制阻断策略. 提出了环境 中耐药基因研究的六大科学前沿, 其中科学前沿中的 前三节是以污水处理厂作为水环境的关键切人点来展 开讨论.

\section{1 科学前沿}

\section{1 耐药基因的主要选择压力: 抗生素的标准检 测体系与共享基础数据库的建立}

目前, 我国抗生素滥用情况严重, 水环境及其相关 环境介质中抗生素残余达到前所未有的高峰, 其造成 
的污染问题也受到了空前的关注. 由抗生素滥用导致 的抗生素污染和耐药性的污染是一个问题的两个方面, 二者相互联系, 不可分割对待. 抗生素被生物体摄人吸 收后, 绝大部分抗生素通过粪便和尿液排出体外进人 环境，造成抗生素在水体和土壤等环境介质中的残留. 抗生素的残留会诱导选择抗性细菌，促进耐药基因的 水平转移, 导致微生物耐药性的扩散, 而携带耐药基因 的微生物扩散到新的环境会进一步繁殖，并有可能通 过基因水平转移将耐药基因传递给病原菌, 给人类健 康带来灾难性的危害. 作为化学污染物, 最近几十年来 抗生素从不同来源, 包括家庭、医院和制药厂, 排放到 工业废水处理厂和城市污水处理厂中. 因此, 全球不同 的工业废水/城市污水处理厂已广泛检测到多类抗生 素 ${ }^{[11]}$. 到目前为止, 已在工业废水/城市污水中检测到的 抗生素包括 $\beta$-内酰胺类、磺胺类、氟喹诺酮类、四环 素类、氨基糖苷类、大环内酯类和糖肽类等 ${ }^{[11]}$. 工业 废水/城市污水处理厂中不同处理单元的抗生素浓度有 很大差异, 有时甚至达到1 2个数量级. 由于抗生素消 费模式、季节性波动等因素，城市污水处理厂进水中 抗生素浓度有着显著的变化. 即使在同一个国家, 不同 地区的抗生素消费模式也有所不同，导致污水处理厂 中的抗生素浓度差异显著. 例如, 在香港的某污水处理 厂进水中检测到低浓度 $(<15 \mathrm{ng} / \mathrm{L})$ 的头孢噻肜，而在邻 近的深圳某污水处理厂进水中检测到的头孢噻肜平均 浓度却高达 $1100 \mathrm{ng} / \mathrm{L}$. 对于头孢氨芐，趋势恰好相反: 深圳某污水处理厂进水中的浓度低于检测限，香港某 污水处理厂进水中的浓度却高达 $1900 \mathrm{ng} / \mathrm{L}$. 其次, 不同 的采样时间也会影响污水中抗生素浓度的检测结果, 因为抗生素使用的季节性波动很大，而且一天中每小 时的浓度都存在着一定的变化 ${ }^{[12]}$. 因此, 不同的采样方 式(随机采样/复合采样、流量/时间比例采样)将得出不 同的结论. 此外, 我们前期研究亦表明城市污水处理厂 平均每日向环境中排放的抗生素总量会因抗生素消费 模式和数量不同而存在地区而异，即使在同一个城市 也是如此 ${ }^{[12]}$, 这主要取决于污水处理厂集水区的人口 规模. 上述结果提示, 要回答耐药性环境污染的第一个 基本科学问题, 即准确定量不同类型环境样品中作为 耐药基因主要选择压力的抗生素的实际残留浓度，以 及全面掌握各热点污染源(包括抗生素制药厂、畜禽 养殖场、屠宰场、医院、污水处理厂等)向环境中排 放抗生素总量的基础数据. 除了精准的分析方法, 还需 要有系统化的采样方案、标准化的采样和预处理流程,
这样才能建立全国尺度乃至全球尺度具有可比性的抗 生素污染水平基础数据库，进而夯实评估抗生素耐药 基因环境选择压力的科学基础. 尽管目前一些国家和 地区已经对污水处理厂中的抗生素残留浓度与种类进 行了大量调查，但系统化、标准化的典型城市污水处 理厂中抗生素大规模长期监测数据仍然十分有限.

由城市污水处理厂推广至水环境, 过去 10 年间, 我 国学者已开展了相关研究工作．Luo等人 ${ }^{[13]}$ 最早对海 河流域抗生素赋存种类、浓度及其环境归趋开展了规 模化的调研, 目标抗生素涵盖四大类12种,研究表明磺 胺氯哒嗪可以作为海河流域畜牧业污染源的指示性污 染物; 河流流量对磺胺类、喹诺酮类和大环内酯类抗 生素浓度的衰减影响最为显著, 而底泥中总有机碳含 量和阳离子交换容量对四环素类抗生素的浓度影响最 大. Zhang等人 ${ }^{[1]}$ 联合采用“流域实地检测 + 药企市场调 查+三级逸度模型模拟”多维度方式，以六大类 36 种抗 生素作为调研目标，对中国抗生素使用量和全国58个 流域抗生素的排放量进行了系统化的研究，成功得到 了包括长江、黄河、珠江等各大流域的抗生素排放量 和排放密度, 准确预测了抗生素在全国58个流域的环 境浓度，揭示了流域范围内医院及水环境样品中细菌 耐药率与该流域范围内抗生素使用量和预测环境浓度 值具有相关性.

在已有工作基础之上，建议今后的重点研究方向 应建立并采用统一的标准检测体系(包括样品采集、 保存、预处理及仪器检测) 定期进行大规模(扩大目标 抗生素检测种类范围)、系统性的流域/区域调查研究 和季节性时空分布特征研究. 水环境样品类型主要涵 盖城市生活污水处理厂系统(进水、泥水混合液、各 工艺出水、最终消毒出水及污泥脱水液)、畜牧养殖 系统(畜禽养殖废水及畜禽粪便)、水产养殖系统(淡水 养殖鱼塘水体及沉积物，近海养殖系统水体及沉积 物)、医院废水、抗生素制药厂废水、垃圾渗滤液及 沥滤液、地表自然水体(河水、湖水和海水)及饮用水 等. 以期建立完善的全国范围各流域中抗生素污染水 平数据库, 且数据定期更新, 以便长期动态跟踪评估抗 生素污染水平变化.

\section{2 水环境中抗生素的迁移转化与生物可利用性}

污水处理过程中抗生素的主要去除途径包括吸 附、生物降解、消毒氧化以及膜分离, 而其他去除途 径, 例如水解、光解和挥发, 考虑到它们在污水处理过 
程中对抗生素的去除比重一般很小，故基本可以排除. 已有的实验室研究结果表明, 生物降解和吸附是活性 污泥法中抗生素的主要去除途径 ${ }^{[14]}$. $\beta$-内酰胺类抗生 素可以通过生物降解去除, 而四环素类抗生素与氟喹 诺酮类抗生素则主要通过活性污泥絮体或生物膜吸附 得以在固相富集, 从而导致污水中浓度快速降低 ${ }^{[14]}$. 需 要注意的是, 尽管此类抗生素在废水中的浓度相对较 低(通常在0.01 1 $\mu \mathrm{g} / \mathrm{L}$ 范围内), 但因其泥-水分配系数 很高, 故在活性污泥或生物膜固相中的浓度可高达 $0.1 \sim 10 \mathrm{mg} / \mathrm{kg}$. 在这种亚抑制浓度下，活性污泥或生物 膜中的细菌不会被杀死或抑制，但在选择压力下有机 会产生或者获得抗性 ${ }^{[15]}$. 目前相关的数据仍十分有限, 需要进一步研究常用抗生素在污水处理过程中和排放 到水环境中之后的生物降解和吸附以及生物可利用性, 为预测这些抗生素在水环境中的迁移转化和生物可利 用性提供基础的科学数据. 抗生素排放到水环境中之 后, 除了生物降解和吸附机制, 水解和光解作用对于抗 生素的迁移转化及其生物可利用性亦具有重要影响. 此外, 经生物或化学转化之后的中间产物及最终产物 的残留抗生素效价亟须量化评估，水环境中长期残留 的多种痕量浓度抗生素及其转化产物因固相富集效应 而引起的环境土著菌群抗生素耐药性的形成、维持及 传播规律值得重点关注和深人研究.

\section{3 耐药基因在污水处理厂生态系统中的转移和 扩散}

活性污泥工艺自1914年被成功开发以来已在全世 界范围内得到了广泛应用, 截至目前已有106年的历史. 该工艺在控制去除常规污染物(包括悬浮固体、五日 生化需氧量/化学需氧量、养分 $\mathrm{N} / \mathrm{P}$ 等)方面发挥着重要 作用. 活性污泥中的细菌多样性非常之高, 在相同的测 序深度下，例如每个样品 17000 条 $16 \mathrm{~S}$ rRNA基因序列, 使用 $97 \%$ 的相似性作为物种水平OTU(操作分类单位) 的划分标准, 污水处理厂活性污泥样品中OTU数目高 达3000余个 ${ }^{[16]}$. 这与土壤样品中的细菌多样性相当, 但 远高于人类肠道中的微生物群落多样性. 此外, 活性污 泥絮体或生物膜通过吸附富集，可使其表面抗生素达 到亚抑制浓度的选择压力, 活性污泥中很高的微生物 多样性和高生物量密度 $(2 \sim 50 \mathrm{~g} / \mathrm{L})$ 、细胞之间很短的 距离和曝气池中泥水的持续摚拌混合，均为抗生素耐 药基因的种内/种间水平转移提供了一个非常有利的重 要生态环境. 值得注意的是, 该生态环境完全是由人工
构建而成, 在自然环境中并不存在. 活性污泥中细菌的 平均繁殖时间(代时)约为 15 30 d, 远快于自然水环境 和土壤中细菌的生长速度, 这就意味着在活性污泥曝 气池这一有利人造生态环境中加速的种内/种间水平基 因转移会进而随细菌的快速传代而被进一步放大. 耐 药细菌会随处理后的污水一起排放至环境当中，从而 增加人类和动物暴露耐药细菌的风险. 每立方米污水 处理厂的出水中通常携带 $10^{8} \sim 10^{9}$ 个细菌, 考虑到目前 常规处理工艺中广泛采用的二沉池并不能完全去除悬 浮物, 故经处理后的大量污水向受纳水体的持续排放, 将不断增加天然水体和沉积物中的耐药基因组. 处理 后的污水如用于农业灌溉, 将会污染土壤. 此外, 经厌 氧消化处理后的剩余活性污泥作为土壤改良剂和肥料 施用时，污泥中的耐药细菌亦会显著增加土壤中的耐 药基因组. 截至2019年2月，我国已累计建成并投人使 用5500余座城市污水处理厂 (不含乡镇污水处理厂和 工业废水处理厂), 污水处理能力达 2.04 亿 $\mathrm{m}^{3} / \mathrm{d}$ (http:// www.water8848.com/news/201908/15/120763.html). 作 为关键的点源污染源, 城市污水处理厂在抗生素耐药 性传播中的重要性不容忽视 ${ }^{[17]}$. 目前, 与其他环境(如 土壤和粪便)中的耐药基因研究相比, 活性污泥和污水 中的耐药基因研究还非常有限 ${ }^{[9]}$, 不足以全面、深人 回答抗生素耐药基因在污水处理厂生态系统中的转移 和扩散这一重要科学问题.

\section{4 探索耐药基因暴露水平研究的标准化方法: 宏基因组学方法和高通量qPCR方法}

过去10余年的前期研究中, 实时定量PCR(qPCR) 曾作为一种主要的方法用于不同环境中 ARGs丰度和 多样性的调查. 然而, 常规的qPCR 方法通量低、效率 低, 可利用的ARGs 引物数量少, 且qPCR结果受多种因 素影响，包括qPCR反应体系、qPCR仪、DNA提取物 的基质效应、操作者的技术水平等. 由于耐药基因是 一个全球性问题，特别是在2013年G8峰会发布了关于 控制抗生素抗性的联合行动声明之后, 科学界和环境 管理层面都希望有更多系统性的可比较的数据集来确 定重要的污染源，比较不同国家和地区间耐药基因的 总体水平差异. 高通量qPCR技术的成功开发可以有效 地解决常规qPCR方法检测通量的问题，目前可检测近 300 种已知的ARGs. Zhu等人 ${ }^{[18,19]}$ 采用该技术开展了 系列研究，对我国3个大型养猪场及其周边地区采集的 样品(包括猪粪、猪粪堆肥和施用堆肥的土壤)进行了 
定量分析．共检测到149种ARGs，其中有63种ARGs丰 度显著高于对照组样品中的丰度. 研究还发现ARGs的 丰度与环境中抗生素和重金属(砷、铜等)浓度呈显著 正相关关系，说明抗生素和重金属的复合污染可能导 致环境中ARGs的富集. 此外, 通过大尺度调研分析了 全国18个主要河流河口沉积物中ARGs的多样性及其 影响因子, 揭示了ARGs的组成与典型抗生素残留浓度 和流域社会经济参数(包括人口和生活污水量等)显著 相关, 表明人类活动(包括养殖业和生活污水排放)是影 响河口ARGs的主要因子.

随着二代/三代高通量DNA测序技术的快速发展， 宏基因组学方法正在成为一种被普遍接受的广谱定量 检测方法. 广泛应用于各种复杂环境样品中ARGs的全 面分析，现在该方法可同时检测涵盖24个类型的 1208 种ARGs亚型 ${ }^{[20]}$. 它不仅可以克服qPCR方法中引物设 计的局限性，基于相似性搜索的基因注释还能给出比 qPCR方法更全面的ARGs的总体情况，而且可以将该 时刻该样品的全部序列存档以备将来对新的耐药基因 的回溯翻查. 利用所建立的宏基因组学检测方法, 本课 题组 ${ }^{[10]}$ 对 10 类典型环境样品开展了耐药基因的广谱解 析研究, 揭示了底泥/土壤/地表水/饮用水-城市污水处 理厂系统-畜禽粪便及养殖场废水处理系统这一全链 条水环境体系中耐药基因丰度分布规律以及耐药基因 的源汇特征(图1). 在这10类典型水环境样品中共检测 到属于 18 种ARGs类型的 260 种ARGs亚型，单个亚型的 丰度范围为 $5.4 \times 10^{-6} \sim 2.2 \times 10^{-1} \mathrm{ARGs}$ 拷贝 $/ 16 \mathrm{~S}-\mathrm{rRNA}$ 基 因拷贝. 不同环境中 ARGs总丰度与这些环境的人为影 响程度十分相关, 从受影响较小的自然环境到严重受 影响的畜禽养殖环境, ARGs总丰度增加了 3 个数量级, 即从 $3.2 \times 10^{-3}$ 升至 3.1 个 ARGs拷贝 $/ 16 \mathrm{~S}-\mathrm{rRNA}$ 基因拷贝。 丰度较高的 $\mathrm{ARGs}$ 主要为氨基糖苷类、杆菌肽、 $\beta$-内 酰胺类、氯霉素类、大环内酯-林可霉素-链阳霉素 类、喹诺酮类、磺胺类和四环素类耐药基因，与广泛 用于人类医疗或动物医疗及生长促进剂的抗生素类型 高度一致. 上述结果充分证实了人类生产活动和抗生 素使用是影响耐药基因在环境中分布和扩散的主要驱 动因子.

高通量qPCR方法优点是检测通量较高、灵敏度 高, 但是仍无法完全克服PCR 扩增过程中的偏差以及 可能的假阴性/假阳性结果, 且只能检测已知ARGs. 相 比于高通量qPCR方法, 宏基因组学方法检测通量更高, 且检测通量随着数据库的扩充可进一步提升, 基于相
似性搜索的宏基因组学方法虽然只能检测已知ARGs, 但对于DNA序列数据集中存在的新型ARGs可进行回 溯翻查, 实现数据的再分析与再挖掘, 检测灵敏度方面, 在现有可接受测序价格的测序深度下，宏基因组学方 法的检测灵敏度逊于高通量qPCR方法. 综上所述, 高 通量qPCR方法和宏基因组学方法各有所长, 互为补充, 可作为探索复杂样品体系中耐药基因暴露水平研究的 备选标准化方法.

ARGs数据库是宏基因组学检测方法的核心所在, 数据库的完整度会显著影响到检测结果的准确性, 即 较低的数据库完整度会低估样品中的ARGs丰度与多 样性，因此使用该方法所面临的关键技术瓶颈是如何 有效、准确地扩充现有的ARGs数据库. 联合应用功能 宏基因组学和基于隐马尔可夫模型预测注释的鉴定方 法, 对新型ARGs进行高通量准确篮查并增加新型 ARGs以提高数据库本身的完整度是当前 ARGs研究领 域的热点之一. 对于高通量qPCR方法而言, 进一步扩 充现有的引物清单, 针对目前ARGs数据库中已有但尚 未包括在高通量 qPCR检测方法引物清单中的耐药基 因参考序列, 设计相应的特异性引物(需全面统筹考虑 引物的完整度、扩增效率和特异性), 是今后重要的科 研攻关方向.

\section{5 耐药基因水平转移和环境传播的关键可移动 遗传元件}

抗生素耐药性的产生可以是原发性，即通过纵向 基因遗传产生, 亦可是获得性产生. 获得性产生耐药性 主要是通过菌株本身DNA的突变或通过细胞间水平基 因转移获得抗性. 在环境领域, 最受关注的是种间的水 平基因转移. 众所周知, 质粒(plasmid)和整合子(integron)等可移动遗传元件在耐药基因的水平转移过程中 发挥了重要作用. 质粒是一种独立于染色体之外的遗 传元件, 具有很强的自主复制和转录能力, 且能在细菌 间发生水平转移，被认为是转移耐药基因最重要的一 类载体. 自20世纪50年代首次发现细菌携带抗性质粒 起, 已从临床菌株和环境菌株中提取到耐受各种抗生 素的质粒，其中在活性污泥体系里分离出的抗性菌携 带的多个质粒上共累计检测到140种耐药基因. 尽管质 粒在耐药基因水平转移的研究中一直受到广泛关 注 $^{[21 ~ 23]}$, 但对质粒携带和转移耐药基因的前期研究大 都是基于质粒分离和捕捉，缺乏对各类菌群携带的质 粒抗性组的全面认识和系统总结. 整合子位于染色 
(a)
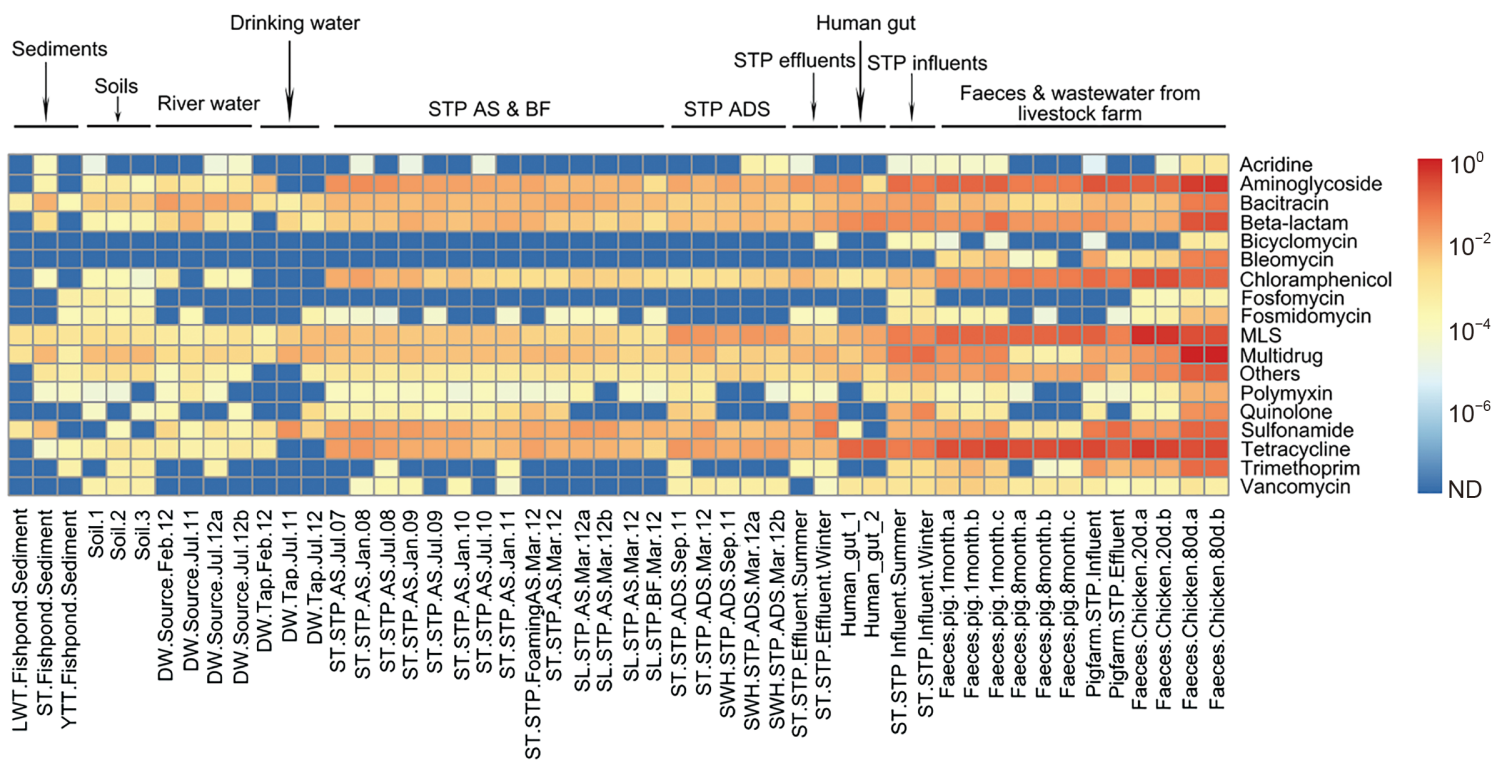

(b) $\stackrel{0}{\frac{1}{0}}$

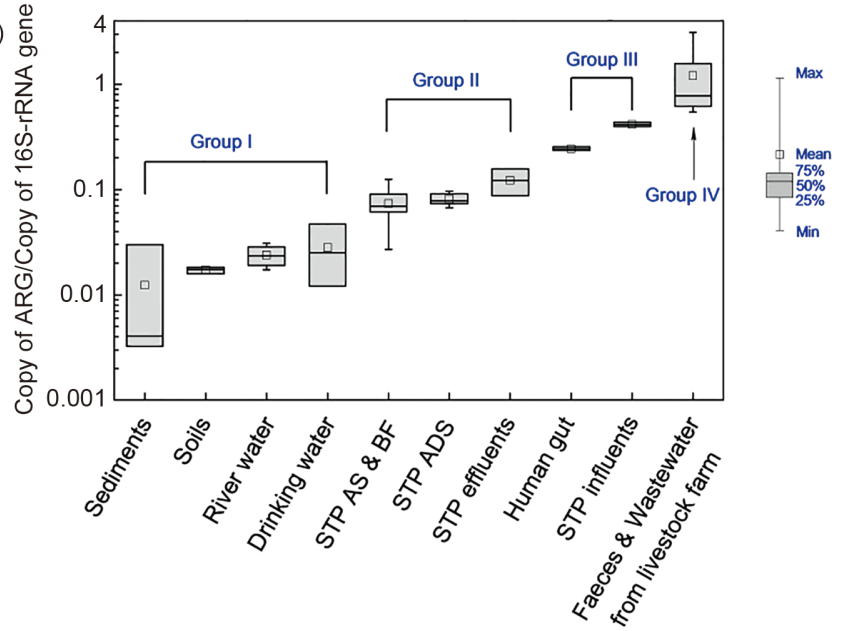

图 1 典型环境样品中耐药基因的赋存状态. (a) 50 个环境样品中抗生素耐药基因的丰度分布(单位: ARGs拷贝数/16S-rRNA基因拷贝数); (b) 不 同类型环境样品间抗生素耐药基因总丰度比较 ${ }^{[8]}$

Figure 1 The occurrence of ARGs in typical environments. (a) Broad-spectrum quantitative profile of the ARGs types (unit: Copy of ARGs per copy of 16S-rRNA gene) in 50 environmental samples. (b) Comparison of total ARGs abundance in different environments ${ }^{[8]}$

体、质粒或转座子上，并通过捕获外源性耐药基因来 增强细菌的耐药性. 本课题组 ${ }^{[24]}$ 前期研究表明, 单个 基因盒可携带多达3类耐药基因类型. 整合子是携带多 种耐药基因的重组表达系统，在多种环境介质中均被 频繁检出，是目前耐药基因水平转移研究领域的一个 重要热点方向. I型整合子在各种环境中最为常见 ${ }^{[25]}$, 广泛存在于微生物的质粒和染色体上，且多数I型整合 子广泛携带耐药基因盒, 可以对多种抗生素具有耐药 性. 目前已发现属于 8 类耐药基因类型的 59 种耐药基因 亚型被I型整合子所携带, 主要包括氨基糖苷类、 $\beta$-内 酰胺类、氯霉素类、大环内酯-林可霉素-链阳霉素
类、喹诺酮类、利福平类、磺胺类和甲氧芐啶类等耐 药基因 ${ }^{[26]}$.

认识环境中耐药基因的传播媒介并阐明其在环境 中的传播机制，是提出有效的耐药基因污染控制阻断 措施的必要前提. 从污染源排放出来的耐药基因的主 要载体是人源菌群(以人类肠道菌为主)和动物源菌群 (以动物肠道菌为主), 这些耐药基因排放到环境之后会 在抗生素和其他环境因子(例如重金属、消毒剂等)的 选择压力下被选择和富集(图2) ${ }^{[27]}$, 并通过水平基因转 移传递给环境土著菌. 环境土著菌作为耐药基因的环 境赋存载体又有机会通过水平转移机制将选择后的耐 


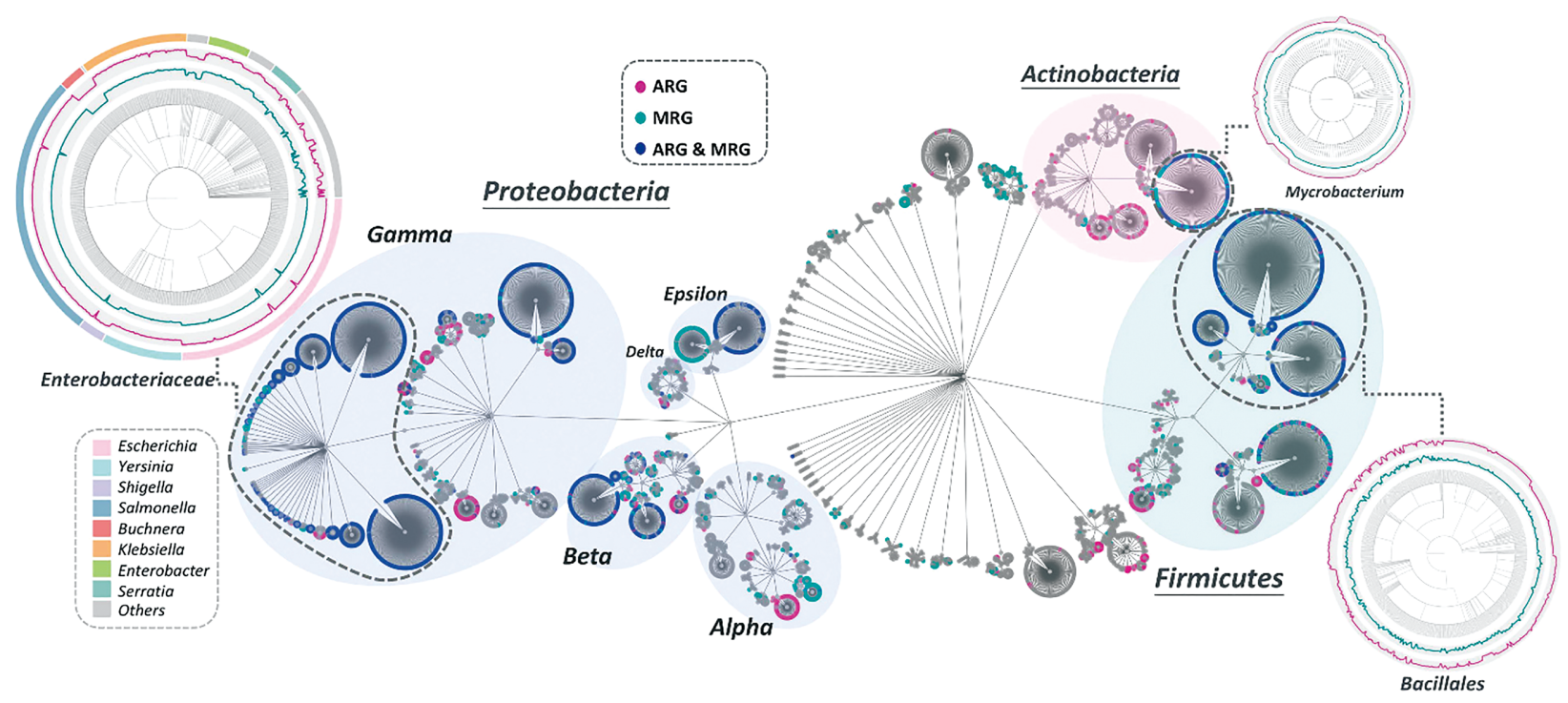

图 2 完整基因组中抗生素耐药基因与重金属抗性基因的赋存特征. 各株菌的系统发育谱系如下: 界、门、纲、目、科、属、株. 携带有抗生 素耐药基因和(或)重金属抗性基因的菌株(灰色节点表示)用不同颜色突出显示. 包括变形菌门、厚壁菌门和放线菌门在内的3个主要的门被突 出显示, 其中携带有抗生素耐药基因和(或)重金属抗性基因的菌株所对应的耐药/抗性基因丰度数据以圈图形式展示(丰度数据经log2转化) ${ }^{[27]}$

Figure 2 Presence and absence of ARGs and MRGs in the complete genome collection. The phylogenic lineages on circular tree for each strain are as follows: domain, phylum, class, order, family, genus and strain. The strain nodes (gray) are highlighted when they carry resistance genes. Three main phyla (Proteobacteria, Firmicutes and Actinobacteira) are highlighted, of which the main groups carrying resistance genes are presented as circles with the ARGs and MRGs abundance profile (log2 transformed) of each genome $\mathrm{e}^{[27]}$

药基因重新传递给人类或动物肠道菌，甚至致病菌(包 括人类致病菌、动物致病菌和人畜共患致病菌)，此类 高风险性耐药基因给人类造成潜在的健康威胁. 由可 移动遗传元件介导的环境土著菌-人类致病菌/动物致 病菌/人畜共患致病菌之间的耐药基因水平基因转移机 制以及高风险耐药菌在环境-动物-人之间的传播机制, 是需要高度关注和深人研究的重点.

\section{6 耐药基因的基因组大数据深度挖掘和机器学习}

高风险性耐药基因的水平基因转移问题是目前耐 药基因相关研究的热点与难点. 前期研究主要基于纯 菌分离培养的方法, 通量有限且无法研究大量的不可 培养的环境土著菌. 细菌培养结合高通量测序和生物 信息学分析的研究方法正在逐步形成并发展完善，以 此为基础的基因组大数据深度挖掘和机器学习有助于 更深人认识耐药基因的水平基因转移规律，从而可进 一步指导科研人员建立起阻断其环境传播的技术手段. 目前, 虽然对水平基因转移有了一些基本的认识, 但缺 乏对耐药基因在不同细菌宿主之间的水平基因转移的 系统性研究和在不同环境条件下水平基因转移速率的 量化评估方法, 无法全面了解其在水环境中的传播和 转移机制. 此外, 耐药基因作为新兴的生物类污染物,
其在复杂环境中的溯源分析亦是科研人员重点关注的 前沿课题. 以基因组大数据为基础的深度数据挖掘和 机器学习人工智能方法(图3), 结合实验室模拟试验和 实际复杂环境大样本调查可以有效地回答上述科学问 题 ${ }^{[28]}$.

\section{2 环境健康风险评估}

抗生素耐药性的风险主要包括两种类型: (1) 医疗 上抗生素治疗失效的风险; (2) 耐药基因在环境中传播 和转移的风险. 人类会通过饮用水、食物或通过直接 接触一些特定环境而暴露于耐药细菌和耐药基因, 从 而导致医疗上抗生素治疗失效的风险. 这种风险的层 级除了取决于暴露水平, 还取决于其他一些因素, 如携 带耐药基因的宿主、暴露途径和致毒因子等. 目前针 对耐药基因和耐药菌的环境风险评价尚缺乏定量模型, 故无法评估复杂环境样品体系中耐药基因的风险，但 普遍的共识是: 除了耐药基因的种类和丰度水平, 还需 要了解这些耐药基因的宿主是否为致病菌 ${ }^{[29]}$, 以及是 否与一些可移动遗传元件关联共存. 前者可以直接决 定抗生素治疗失效的风险，而后者会影响一个耐药基 因在环境中传播和转移的机率, 从而间接决定抗生素 治疗失效的风险. 鉴于此, 基于长读长的三代基因测序 

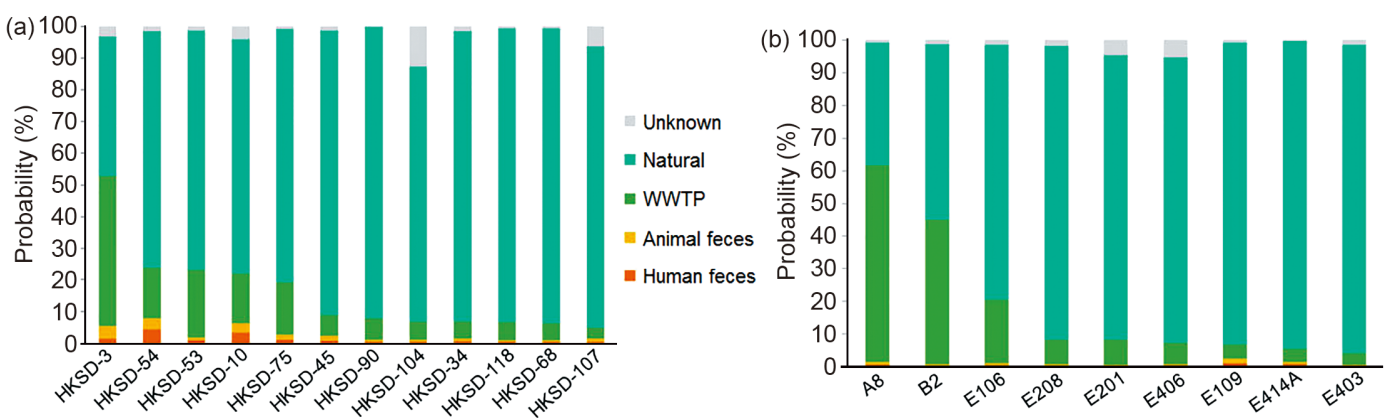

图 3 抗生素耐药基因污染来源贡献百分比估计值. (a) 香港底泥样品; (b) 珠江口与南海底泥样品 ${ }^{[28]}$

Figure 3 The predicted source proportion of Hong Kong sediment samples (a) and Pearl River Estuary and South China Sea sediment samples (b) ${ }^{[28]}$

是极为有效的手段(图4), 可以准确鉴定耐药基因的携 带宿主及其所处的遗传元件定位，从而可用来帮助科 研人员评估抗生素耐药基因的风险层级 ${ }^{[23]}$.

一些环境污染控制工程单元和再生水/固废处置环 节, 如制药废水生物处理反应器、城市污水生物处理反 应器、污泥作为土壤改良剂和肥料、再生水回灌等, 都 会在某种程度上增加耐药基因在环境中传播和转移的 风险 ${ }^{[30-33]}$. 除此之外, 近年来也有报道指出,一些非抗生 素类污染物如重金属 ${ }^{[34]}$ 和合成化合物如三氯生 ${ }^{[35]}$ 均可 被活性污泥或生物膜吸附, 通过共选择机制形成对耐药 基因的选择压力而促进了耐药基因的富集、转移和扩 散. 因此, 在评估耐药基因环境健康风险时, 除了考虑耐 药基因的种类、丰度水平、可移动性、耐药基因宿主 种类及致毒因子, 还需要综合考量该环境体系中共存的 重金属污染物和其他合成化合物等可形成耐药基因选 择压力的因素影响. 此外, 各因素所占权重如何合理确 定亦是构建环境健康风险评估定量模型的难点.

根据“卫生一体化”(One Health，又称“同一个健 康”) 的理念, 环境健康、动物健康与人体健康三者紧 密联系, 相互影响, 任何单一要素均无法做到“独善其

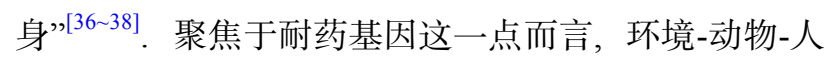
三个要素之间, 尤其是在全球尺度下微生物及其携带 的耐药基因受人类活动显著影响而发生大规模迁徙的 进程中 ${ }^{[39]}$, 环境中的耐药基因和耐药菌对人体健康的 影响究竟起多大作用? 环境中的耐药基因和耐药菌通 过不同的媒介方式传播给人体的比重有多大? 这些科 学问题均是目前研究的重中之重. 现有研究多侧重于 大规模调研分析环境样品(土壤、污水、人和畜禽粪 便等)和人类致病菌/人体肠道微生物共有的耐药基因 并做同源性分析 ${ }^{[40,41]}$, 或者通过多变量逻辑回归分析 模型分析社会经济参数(不同区域的水产养殖发达程
度、日人均水产品的摄入量、年人均猪肉、羊肉、总 肉类的消费量等)与携带 $m c r-1$ 基因大肠杆菌的显著相 关性等方法 ${ }^{[42]}$ ，间接揭示环境中耐药基因和耐药菌对 人类健康的潜在影响. Sun等人 ${ }^{[43]}$ 采集了 14 名学生在养 猪场实习前、实习期间及返校后的粪便样品、养猪场 工人粪便样品、猪粪便样品及养猪场环境样品(土 壤、污水和通风(尘埃), 通过联合应用 $16 \mathrm{~S}$ rRNA扩增子 测序、宏基因组学和培养组学分析手段, 发现养猪场 环境可在短期内改变实习学生肠道菌群和耐药基因组 成. 该研究首次提供直接证据证实了环境中的微生物 及其携带的耐药基因可传播至人体肠道. 综上所述, 后 续研究需提供更多直接证据并重点关注: (1) 量化环境 中的耐药基因和耐药菌通过不同的媒介方式传播给人 体的比重; (2) 量化环境中的耐药基因和耐药菌对人体 健康的影响.

\section{3 防控阻断策略}

耐药性的防控阻断策略包括政策管理和工程技术 两大方面. 在政策管理方面, 涉及多学科联动, 需要临 床医学、生物学、生态学、化学、农学、药学、环境 科学与工程等多方面的共同努力, 还需要法律学、社 会学、应用经济学和教育学等方面的配合. 水环境中 抗生素与抗生素抗性污染防控应树立并坚持“One Health”全局式理念，主动纳人到《遏制细菌耐药国家 行动计划(2016 2020年)》的框架之下. 在工程技术方 面，虽然污水处理、固废处置和医疗上的抗生素治疗 失效并非直接相关，或许有些人会质疑在这方面加大 投人进行防控阻断的实际效益，但是从“卫生一体化” 的角度来看，如果不在这些关键节点上投人资金减少 抗生素和耐药基因的环境排放，以后就要在医院和社 区投人更多的资金来被动应对因环境暴露而引起的耐 


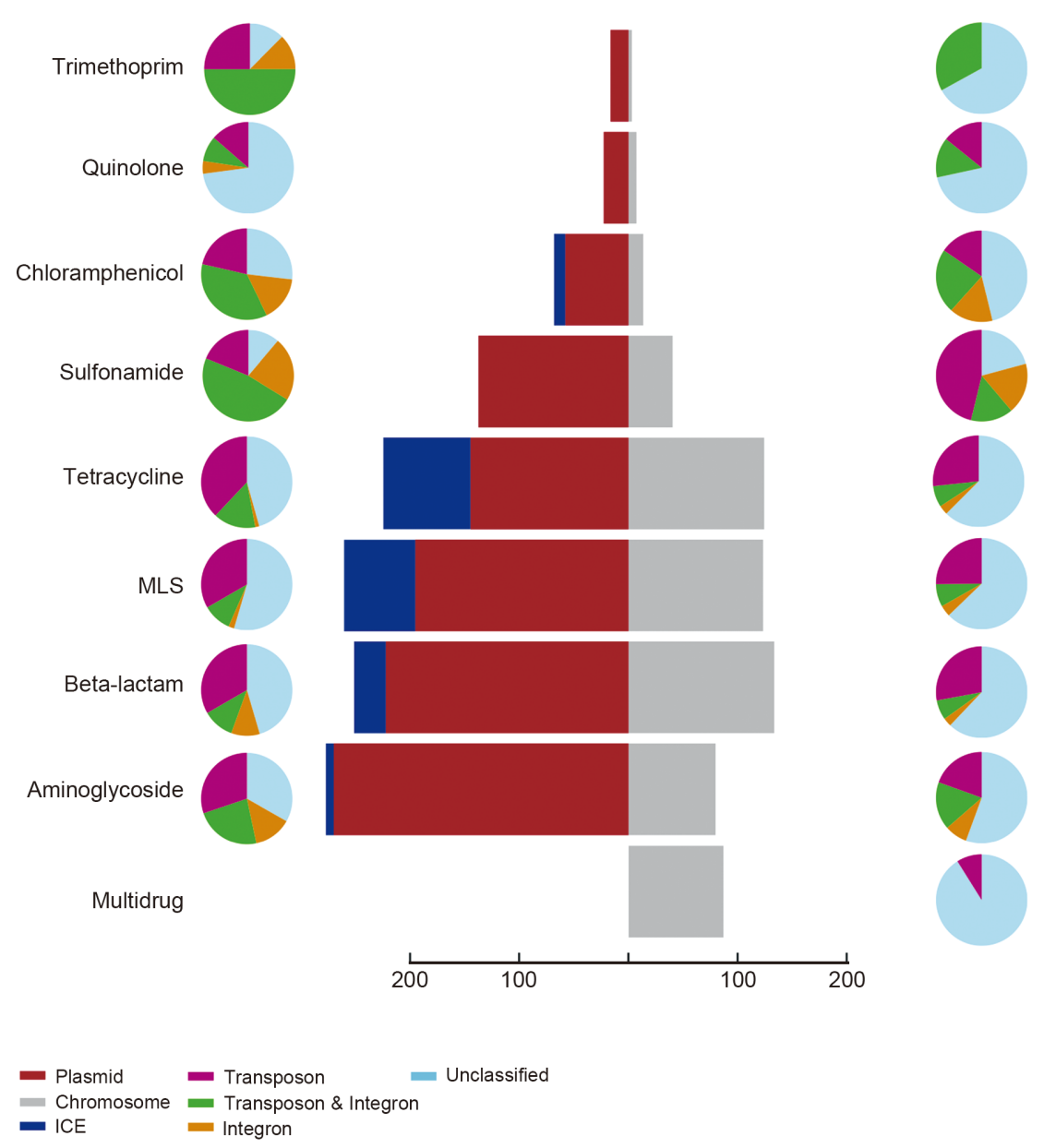

图 4 应用纳米孔宏基因组数据集分析预测环境样本(污水厂进水、活性污泥和出水)中抗生素耐药基因所在基因元件位置. 其中, 抗生素耐药 基因分布于 1791 条纳米孔长序列上. 柱状图表示抗生素耐药基因在质粒、整合与接合元件以及染色体上的分布与丰度; 饼图(左)表示质粒上携 带抗生素耐药基因的转座子和整合子的分布模式; 饼图(右)表示染色体上携带抗生素耐药基因的转座子和整合子的分布模式 ${ }^{[23]}$

Figure 4 Genetic location of antibiotic resistance genes (ARGs) predicted from the nine Nanopore metagenomic datasets of environmental samples including influents, activated sludge and effluents. The resistome was presented by a total of 1791 ARGs-carrying long reads. Bar chart: Distribution and abundance of ARGs carried by plasmids, integrative and conjugative elements (ICEs) and chromosome; pie chart: Distribution pattern of transposons and integrons located on plasmids (left) and chromosome carrying reads (right) ${ }^{[23]}$

药性危机. 目前仍然不可能大幅度改造提升现有的城 市污水及固废处理工艺与设施，以提高对这些新兴污 染物的去除效率, 减少和完全阻断其环境排放. 但是从 工程实施和运行管理的角度来看, 可以优先采取效益 成本比高的措施来防控和阻断耐药基因的污染. 尽管 仍然缺乏系统性的数据支持, 但根据帕累托法则, 可以 合理地推断出需要对如下所列重点污染源优先采取防 控阻断措施：医院废水、制药废水以及畜禽养殖(包括 水产养殖)粪便/废渣及其污水等. 对于医院废水，在将 其排人与城市污水处理厂相连的市政污水管网之前, 应该用膜分离技术中的超滤膜去除病毒和细菌，也可 以考虑用纳滤膜去除废水中的化学污染物(抗生素本
身和其他类药物) 以及携带耐药基因的游离DNA片段. 对于制药废水, 在直接排人市政污水管网之前, 务必进 行处理, 但强烈建议不要继续采用现有的传统二级生 物处理工艺, 因为那可能是超级耐药细菌的孵化器. 如 果无法避免生物处理或对已经采用了生物处理的设施, 应加装膜分离装置, 将细菌隔离在制药废水设施之内 而不外排. 对于来自上述重要污染源的污泥应该以焚 烧为最终处置方式, 而不应再考虑其他处置方法, 无论 是填埋还是作为肥料施用(不管是农用地还是非农用 地)都应该避免. 对于畜牧业的牲畜粪便, 则应考虑以厌 氧发酵处理 ${ }^{[44,45]}$ 或将其转化成生物碳的方式进行控制, 阻断其向环境的传播. 


\section{参考文献}

1 Zhang Q Q, Ying G G, Pan C G, et al. Comprehensive evaluation of antibiotics emission and fate in the river basins of China: Source analysis, multimedia modeling, and linkage to bacterial resistance. Environ Sci Technol, 2015, 49: 6772-6782

2 O'Neill J. Antimicrobial Resistance: Tackling a Crisis for the Health and Wealth of Nations. 2014, https://amr-review.org/sites/default/files/AMR\% 20Review\%20Paper\%20-\%20Tackling\%20a\%20crisis\%20for\%20the\%20health\%20and\%20wealth\%20of\%20nations_1.pdf

3 Laxminarayan R, Duse A, Wattal C, et al. Antibiotic resistance-The need for global solutions. Lancet Infect Dis, 2013, 13: 1057-1098

4 Willetts D, Livanov D, Schütte G, et al. G8 Science Ministers Statement. London, UK, 2013

5 The White House. National Strategy for Combating Antibiotic-Resistant Bacteria. Washington DC: The White House, 2014

6 Cytryn E. The soil resistome: The anthropogenic, the native, and the unknown. Soil Biol Biochem, 2013, 63: 18-23

7 Pruden A, Arabi M, Storteboom H N. Correlation between upstream human activities and riverine antibiotic resistance genes. Environ Sci Technol, 2012, 46: 11541-11549

8 Li B, Yang Y, Ma L, et al. Metagenomic and network analysis reveal wide distribution and co-occurrence of environmental antibiotic resistance genes. ISME J, 2015, 9: 2490-2502

9 Yang Y, Li B, Zou S, et al. Fate of antibiotic resistance genes in sewage treatment plant revealed by metagenomic approach. Water Res, 2014, 62: $97-106$

10 Ma L, Li B, Jiang X T, et al. Catalogue of antibiotic resistome and host-tracking in drinking water deciphered by a large scale survey. Microbiome, 2017, 5: 154

11 Zhang T, Li B. Occurrence, transformation, and fate of antibiotics in municipal wastewater treatment plants. Crit Rev Environ Sci Technol, 2011, 41: 951-998

12 Li B, Zhang T. Mass flows and removal of antibiotics in two municipal wastewater treatment plants. Chemosphere, 2011, 83: 1284-1289

13 Luo Y, Xu L, Rysz M, et al. Occurrence and transport of tetracycline, sulfonamide, quinolone, and macrolide antibiotics in the Haihe River Basin, China. Environ Sci Technol, 2011, 45: 1827-1833

14 Li B, Zhang T. Biodegradation and adsorption of antibiotics in the activated sludge process. Environ Sci Technol, 2010, 44: 3468-3473

15 Berendonk T U, Manaia C M, Merlin C, et al. Tackling antibiotic resistance: The environmental framework. Nat Rev Microbiol, 2015, 13: 310-317

16 Zhang T, Shao M F, Ye L. 454 Pyrosequencing reveals bacterial diversity of activated sludge from 14 sewage treatment plants. ISME J, 2012, 6: $1137-1147$

17 Czekalski N, Gascón Díez E, Bürgmann H. Wastewater as a point source of antibiotic-resistance genes in the sediment of a freshwater lake. ISME J, 2014, 8: 1381-1390

18 Zhu Y G, Johnson T A, Su J Q, et al. Diverse and abundant antibiotic resistance genes in Chinese swine farms. Proc Natl Acad Sci USA, 2013, 110: $3435-3440$

19 Zhu Y G, Zhao Y, Li B, et al. Continental-scale pollution of estuaries with antibiotic resistance genes. Nat Microbiol, 2017, 2: 16270

20 Yin X L, Jiang X T, Chai B L, et al. ARGs-OAP v2.0 with an expanded SARG database and Hidden Markov Models for enhancement characterization and quantification of antibiotic resistance genes in environmental metagenomes. Bioinformatics, 2018, 34: 2263-2270

21 Zhang T, Zhang X X, Ye L. Plasmid metagenome reveals high levels of antibiotic resistance genes and mobile genetic elements in activated sludge. PLoS One, 2011, 6: e26041

22 Li AD, Li LG, Zhang T. Exploring antibiotic resistance genes and metal resistance genes in plasmid metagenomes from wastewater treatment plants. Front Microbiol, 2015, 6: 1025

23 Che Y, Xia Y, Liu L, et al. Mobile antibiotic resistome in wastewater treatment plants revealed by Nanopore metagenomic sequencing. Microbiome, 2019, 7: 44

24 Ma L, Li A D, Yin X L, et al. The prevalence of integrons as the carrier of antibiotic resistance genes in natural and man-made environments. Environ Sci Technol, 2017, 51: 5721-5728

25 Zhang X X, Zhang T, Zhang M, et al. Characterization and quantification of class 1 integrons and associated gene cassettes in sewage treatment plants. Appl Microbiol Biotechnol, 2009, 82: 1169-1177

26 Zhang A N, Li L G, Ma L, et al. Conserved phylogenetic distribution and limited antibiotic resistance of class 1 integrons revealed by assessing the bacterial genome and plasmid collection. Microbiome, 2018, 6: 130

27 Li L G, Xia Y, Zhang T. Co-occurrence of antibiotic and metal resistance genes revealed in complete genome collection. ISME J, 2017, 11: 651662

28 Li L G, Yin X, Zhang T. Tracking antibiotic resistance gene pollution from different sources using machine-learning classification. Microbiome, 2018, 6: 93 
29 Ma LP, Xia Y, LI B, et al. Metagenomic assembly reveals hosts of antibiotic resistance genes and the shared resistome in pig, chicken and human feces. Environ Sci Technol, 2015, 50: 420-427

30 Liu M, Ding R, Zhang Y, et al. Abundance and distribution of Macrolide-Lincosamide-Streptogramin resistance genes in an anaerobic-aerobic system treating spiramycin production wastewater. Water Res, 2014, 63: 33-41

31 Su J Q, Wei B, Ou-Yang W Y, et al. Antibiotic resistome and its association with bacterial communities during sewage sludge composting. Environ Sci Technol, 2015, 49: 7356-7363

32 Wang F H, Qiao M, Su J Q, et al. High Throughput profiling of antibiotic resistance genes in urban park soils with reclaimed water irrigation. Environ Sci Technol, 2014, 48: 9079-9085

33 Zhang Y, Yang M, Wang C Y. Antibiotic, antibiotic resistance genes, pollutant discharge characteristics, horizontal transfer mechanism, pollution control technology (in Chinese). Environ Chem, 2015, 34: 1-8 [张昱, 杨敏, 王春艳. 生产过程中抗生素与抗药基因的排放特征, 环境行为及控 制. 环境化学, 2015, 34: 1-8]

34 Seiler C, Berendonk T U. Heavy metal driven co-selection of antibiotic resistance in soil and water bodies impacted by agriculture and aquaculture. Front Microbio, 2012, 3: 399

35 Pal C, Bengtsson-Palme J, Kristiansson E, et al. Co-occurrence of resistance genes to antibiotics, biocides and metals reveals novel insights into their co-selection potential. BMC Genom, 2015, 16: 964

36 Collignon P, McEwen S. One Health-Its importance in helping to better control antimicrobial resistance. Trop Med Int Health, 2019, 4: 22

37 McEwen S A, Collignon P J. Antimicrobial resistance: A One Health perspective. Microbiol Spectr, 2017, 6: 255-260

38 Hernando-Amado S, Coque T M, Baquero F, et al. Defining and combating antibiotic resistance from One Health and Global Health perspectives. Nat Microbiol, 2019, 4: 1432-1442

39 Zhu Y G, Gillings M, Simonet P, et al. Microbial mass movements. Science, 2017, 357: 1099-1100

40 Forsberg K J, Reyes A, Wang B, et al. The shared antibiotic resistome of soil bacteria and human pathogens. Science, 2012, 337: 1107-1111

41 Wang Y, Zhang R, Li J, et al. Comprehensive resistome analysis reveals the prevalence of NDM and MCR-1 in Chinese poultry production. Nat Microbiol, 2017, 2: 16260

42 Shen Y, Zhou H, Xu J, et al. Anthropogenic and environmental factors associated with high incidence of MCR-1 carriage in humans across China. Nat Microbiol, 2018, 3: 1054-1062

43 Sun J, Liao X P, D’Souza A W, et al. Environmental remodeling of human gut microbiota and antibiotic resistome in livestock farms. Nat Commun, 2020, 11: 1427

$44 \mathrm{Ju} \mathrm{F}$, Li B, Ma L, et al. Antibiotic resistance genes and human bacterial pathogens: Co-occurrence, removal, and enrichment in municipal sewage sludge digesters. Water Res, 2015, 91: 1-10

45 Zhang T, Yang Y, Pruden A. Effect of temperature on removal of antibiotic resistance genes by anaerobic digestion of activated sludge revealed by metagenomic approach. Appl Microbiol Biotechnol, 2015, 99: 7771-7779 


\title{
Antibiotic resistance in water environment: Frontiers of fundamental research, risk assessment and control strategies
}

\author{
Tong Zhang ${ }^{1 *} \&$ Bing $\mathrm{Li}^{2}$ \\ ${ }^{1}$ Environmental Microbiome Engineering and Biotechnology Laboratory, Department of Civil Engineering, The University of Hong Kong, Hong Kong, \\ China; \\ ${ }^{2}$ Institute of Environmental Engineering and Management, Tsinghua Shenzhen International Graduate School, Tsinghua University, Shenzhen 518055, \\ China \\ * Corresponding author, E-mail: zhangt@hku.hk
}

Antibiotic resistance has been considered as a major health security challenge of the 21 st century in the 2013 G8 Science Minsters Statement. UN Environment ranked "Antimicrobial Resistance: Investigating the Environmental Dimension" as No.1 emerging issue among the six emerging issues of environmental concern in 2017. It should be pointed out that antibiotic resistance is not just a regional or national phenomenon but also a global problem, indicated by the famously typical cases of the rapid dissemination of Klebsiella pneumonia carbapenemase-positive bacteria and New Delhi metallo$\beta$-lactamase-positive bacteria in Asia, Europe and North America. Animal waste, antibiotic production wastewater, hospital wastewater, sewage, sludge, soil, sediment, surface water and even air have been considered as important reservoirs for antibiotic resistance genes (ARGs) because abundant ARGs have been frequently detected in these environments. Among the different environment types, the water environment is a key node for the dissemination of antibiotic resistance. A large number of previous studies mainly focused on the abundance and diversity of ARGs in the water environments. However, the systematic and in-depth studies on the source, spread and fate of ARGs in the water environment are still limited. The basic framework and sophisticated models for risk assessment are still lacking for environments even possessing data of antibiotic concentration and exposure levels of ARGs. In addition, there is also no strategic thinking in the formulation and implementation of antibiotic resistance control strategies. Considering the above issues, this review will focus on the frontiers of fundamental research, risk assessment, and control strategies of antibiotic resistance in water environment and other environments, including: (1) The establishment of standardized detection approach and public database for antibiotics, i.e., the major selection pressure of antibiotic resistance; (2) the migration, transformation and bioavailability of antibiotics; (3) the transfer and dissemination of ARGs in the wastewater treatment plants; (4) exploring the standardized methods for investigating exposure levels of ARGs: metagenomic approach \& highthroughput qPCR approach; (5) the key mobile genetic elements responsible for horizontal gene transfer and environmental transmission of ARGs; (6) deep mining and machine learning of ARGs using metagenomic/genomic big data. Several case studies were selected respectively to demonstrate current research status of the above six frontiers of fundamental research, risk assessment, and control strategies of antibiotic resistance. For the risk assessment, two types of risks including the risk of antibiotic treatment failure and the risk of ARGs dissemination in the environment should be distinguished. The prevention and control strategy of antibiotic resistance involves multi-disciplinary linkage and combination together, which requires the joint efforts of clinical medicine, ecology, agriculture, pharmacy, environmental science and engineering, as well as the cooperation of education, etc. Currently, following the integrative strategy of One Health, we should give the first priority to the investment on prevention and control measures at the key emission sources and other nodes to effectively reduce and ultimately stop the emissions of antibiotics and ARGs. The key emission sources mainly include antibiotic manufacturing factory, livestock farm, aquaculture farms, slaughter house, hospital and municipal wastewater treatment plants etc.

antibiotic resistance genes, water environment, horizontal gene transfer, risk assessment, control strategy

doi: 10.1360/TB-2020-0110 